\title{
Urgences
}

\section{Les chemins perdus : figurations de la vie, de la mort et de la renaissance chez Washington Irving, Alejo Carpentier et Gabrielle Roy}

\section{Jean Morency}

Numéro 34, décembre 1991

Mythes et Romans de l'Amérique

URI : https://id.erudit.org/iderudit/025686ar

DOI : https://doi.org/10.7202/025686ar

Aller au sommaire du numéro

Éditeur(s)

Urgences

ISSN

0226-9554 (imprimé)

1927-3924 (numérique)

Découvrir la revue

Citer cet article

Morency, J. (1991). Les chemins perdus : figurations de la vie, de la mort et de la renaissance chez Washington Irving, Alejo Carpentier et Gabrielle Roy.

Urgences, (34), 75-87. https://doi.org/10.7202/025686ar d'utilisation que vous pouvez consulter en ligne. 


\section{Les chemins perdus: figurations de la vie, de la mort et de la renaissance chez Washington Irving, Alejo Carpentier et Gabrielle Roy}

Jean Morency

En dépit du foisonnement de l'expression littéraire et de la multiplicité de ses formes sur le continent américain, la comparaison des littératures américaines n'acquiert peut-être tout son sens créateur que si l'on accepte de prendre en considération, ne serait-ce que spéculativement, une possible unité qui traverserait, en les reliant, les imaginaires collectifs en terre d'Amérique, par delà les clivages sociaux et culturels, unité qui serait à rechercher, selon moi, du côté de l'idée même du Nouveau Monde telle qu'elle se manifeste en Amérique, du Brésil aux États-Unis, de l'Argentine au Canada, du Chili au Québec; car ce n'est finalement jamais rien d'autre que cette idée, héritée de la Renaissance européenne et qui fut naturellement récupérée par les peuples américains, qui se dévoile dans la diversité des manifestations traduisant ce qu'on nomme l'américanité.

On retrouve en effet, au fondement même de l'aventure américaine, une ambition, secrète ou avouée, qui est celle du recommencement, de la transformation, de la métamorphose, ambition qui demeure sans cesse en butte aux obstacles d'une réalité objective avec laquelle il faut bien composer, sous peine de schizophrénie ou de mythomanie. Si l'*américanité * existe - ce qui reste toujours à prouver en-dehors des conditionnements qui président à l'apparition et à l'affirmation des sociétés américaines et des littératures nationales qui renvoient à celles-ci, plus ou moins déformée, leur propre image - elle serait donc à rechercher du côté des figurations, littéraires ou filmiques, de cette volonté partagée de recommencement absolu. À cet égard, il me semble intéressant d'esquisser ici une tentative de comparaison de trois écrivains "américains " appartenant à des époques et des cultures distinctes, voire même à des * sphères * idéologiques opposées; ces écrivains sont Washington Irving, Alejo Carpentier et Gabrielle Roy. La méthode que je compte utiliser, très 
librement du reste, se fonde sur l'analyse des scénarios mythiques et des éléments significatifs qui contribuent à leur charge sémantique et à leur dramatisation: si effectivement le mythe d'une renaissance constitue le dénominateur commun des imaginaires américains, on devrait retrouver, du moins théoriquement, des reliquats de ce mythe transformationnel et de ses composantes dynamiques à l'intérieur des documents étudiés.

\section{Rip Van Winkle et la (re)naissance de l'Amórique}

Dans cette perspective conjointe de la constitution des littératures nationales et de la mise en forme des figurations imaginaires visant à exprimer la quintessence de l'expérience américaine, le cas de l'écrivain états-unien Washington Irving (1783-1859) s'avère assez éclairant, en ceci que ce dernier prend place dans une culture nationale encore assez mal définie qui se dégage à grand peine de l'emprise coloniale, et à l'intérieur de laquelle la question de la littérature nationale commence déjà à se poser (cette question deviendra plus épineuse aux environs de 1850 et jouera du reste un rôle certain dans la composition de Moby Dick, roman *américain * par excellence). Il est d'ailleurs intéressant de noter que Washington Irving se trouve comme placé au point de confluence d'une réalité fascinante mais obligée - l'Amérique et d'une culture dominante, bien que pleinement assumée celle de l'Europe (où il séjournait d'ailleurs au moment de la rédaction de son fameux Sketch Book, préfigurant en cela la lignée de la *génération perdue*) - et qu'il semble particulièrement sensible à la dualité de sa position, comme l'observe Marc Saporta dans son Histoire du roman américain: * nul n'est plus sollicité par cette quête de l'identité américaine, parallèle à la réception de sources d'inspiration étrangère, que Washington Irving * 1 .

Cette dualité de l'esprit, voire de l'imaginaire tout entier, serait-elle au fondement de ce qu'on nomme, à défaut d'une meilleure appellation, l'américanité, qui ne peut se concevoir sans son contraire? Quoi qu'il en soit, avec son conte le plus célèbre, * Rip Van Winkle (1819), Washington Irving donne 
une impulsion décisive à la littérature états-unienne. L'histoire de Rip Van Winkle, le traîne-savates qui quitte son village de la vallée de l'Hudson pour aller se ballader dans les monts Kaatskills et n'en redescendre que vingt ans plus tard, au lendemain de la Révolution américaine, est assez connue pour qu'il ne soit pas indispensable de la raconter dans tous ses détails. Ce qu'il faut surtout considérer ici, en marge du mystérieux sommeil dans lequel est plongé le héros du conte et qui coïncide avec l'apparition de la nation américaine, ce sont les modalités typiquement initiatiques qui caractérisent l'aventure de Rip Van Winkle et la relient manifestement au scénario paradigmatique de la vie, de la mort et de la renaissance qui gouverne souvent les rituels de passage, tels que les dévoilent les historiens des religions: depuis le mystérieux donateur (au sens où l'entend Vladimir Propp) que Rip Van Winkle croise dans la montagne jusqu'au cirque rocheux où il se trouve entraîné, en passant par les nombreuses figurations métaphoriques du corps féminin rencontrées en chemin, c'est au récit d'une transformation ontologique radicale que Washington Irving convie son lecteur. Voyage paradoxal que celui entrepris sur les hauteurs des Kaatskills, aux confins du fameux « wilderness " américain, et qui conduit finalement Rip Van Winkle au fond d'un vallon presque clos sur luimême, au terme d'une remontée inconsciente vers le lieu de sa propre naissance:

S'assistant l'un l'autre, ils se hissèrent jusqu'au haut d'un étroit ravin apparemment formé par le lit asséché d'un torrent de montagne. Pendant leur ascension, Rip entendait de temps a autre de longs grondements, comme d'un tonnerre lointain, qui semblaient provenir d'un profond ravin, ou plutôt d'une profonde crevasse s'ouvrant entre deux rochers élevés, vers lesquels menait leur chemin rocailleux [...] Ayant traversé le ravin, ils parvinrent à une excavation pareille à un petit amphithéâtre, entourée d'escarpements à pic au bord desquels des arbres suspendus jetaient leurs branches, de sorte que l'on n'y voyait que quelques échappées de ciel azuré et d'éclatants nuages du soir. ${ }^{2}$

2 Washington Irving, Contes fantastiques: "Rip Van Winkle allemand ", " Le gouverneur des sept cités introduction de Roger Asselineau, Paris, Aubier, 1979, p. 171, 173. 
Cette remontée nécessaire vers le lieu de toute naissance, au ccur de toutes choses, au point précis où la vie et la mort s'entremêlent, pourrait être la première image forte de l'américanité: en s'engageant sur le chemin du continent, c'est-à-dire de la régénération, l'homme renonce inconsciemment à sa vie; il doit mourir pour renaître américain. Le long sommeil de Rip Van Winkle constitue ainsi une figuration euphémisante de la mort, en lui, de tout ce qui appartient au monde ancien, tandis que son réveil coïncide avec la révolution américaine, ce en quoi force est d'admettre le génie littéraire de Washington Irving, qui a su faire d'un simple personnage le bouc émissaire d'une société en crise, le médiateur indispensable à une transformation radicale de celle-ci. Mais du même coup, le rapport que ce personnage entretient avec le réel, et partant avec lui-même, se trouve anéanti: en se réveillant, Rip Van Winkle ne se reconnaît qu'à peine, ne retrouve plus son chien ni sa carabine, ni même l'entrée de l'amphithéâtre. Le chemin est perdu à jamais: "Finalement, il parvint à l'endroit où le ravin s'était ouvert à travers les falaises de l'amphithéâtre; mais, de cette ouverture, nulle trace ne subsistait * (p. 181).

Voilà donc la seconde image forte de l'américanité : la perte absolue et inéluctable des points de repère, tant géographiques que temporels et humains, la perte de soi, des racines, des signes rassurants du passé, au terme d'une métamorphose avortée qui ne peut jamais être répétée. En retournant au village, Rip Van Winkle est littéralement désorienté, ne reconnaissant plus rien ni personne, et à plus forte raison lui-même: "Je ne suis pas moi-même. Je suis quelqu'un d'autre [...] J'étais moi-même hier soir, mais je me suis endormi dans la montagne et l'on m'a changé mon fusil, et tout est changé et je suis changé, et je ne peux dire quel est mon nom, ni qui je suis * (p. 195). Cette fracture de la psyché illustre assez bien la portée singulière de l'initiation au monde américain, qui n'intègre jamais l'individu au clan, comme c'est le cas par exemple dans les sociétés traditionnelles, mais le détache de la société pour l'abandonner dans le désert splendide de sa liberté et de sa solitude infinies. Finalement, Rip Van Winkle n'est plus rattaché à la famille et au clan que de manière ironique (comme le suggère l'emploi, dans son patronyme, de l'élément de liaison "van "); 
il est coupé de tous, et surtout de lui-même (en langue anglaise, "rip " signifie d'ailleurs «déchirure»). C'est ainsi que Bertrand Terramorsi écrit :

Rip le déchiré devient la figure primitive emblématique de l'imaginaire schizoïde américain. Il est certes le héros du rêve américain, mais il est aussi l'incarnation du déchirement initial, d'une histoire décousue [...] Rip incarne bien [...] le déchirement radical que provoque la re-naissance du nouvel homme américain. ${ }^{3}$

\section{Los pasos perdidos. Une autre Amérique ?}

La nostalgie inconsciente d'un passé pour jamais aboli, qui pousse Rip Van Winkle vers la montagne sauvage et l'univers des anciens, devient manifeste dans Los pasos perdidos, roman du Cubain Alejo Carpentier paru en 1955 et traduit en français en 1956 sous le titre Le partage des eaux. Certes, il serait illusoire de comparer deux écrivains aussi dissemblables que Washington Irving et Alejo Carpentier, tant chacun semble résumer l'esprit de son temps, si l'on ne prenait en considération leur origine géographique commune et surtout cette manière analogue qu'ils ont de considérer et de mettre en forme la problématique du temps et de l'espace. Suivant en quelque sorte l'exemple de Rip Van Winkle, le narrateur du Partage des eaux rompt avec la société moderne, associée au mythe de Sisyphe et symbolisée par le New-York du milieu du vingtième siècle, pour remonter l'Orénoque à la recherche d'instruments de musique très anciens. Derrière ce voyage dans l'espace se profile bientôt une véritable odyssée dans le temps, qui permet au narrateur de renouer, à rebours du modernisme, avec la splendeur baroque des temps anciens. Cette exploration du passé se fait progressivement: la redécouverte de la langue maternelle (l'espagnol) et des sensations oubliées de l'enfance (notamment des odeurs) enclenche le mouvement inéluctable du retour vers le passé et prélude à la découverte de ce qui se trouve en amont même de la vie:

3 Bertrand Terramorsi, * Le rève américain; note sur le fantastique et la renaissance aux États-Unis ", Europe, $n^{\circ} 707$, mars 1988, p. 16. 
80

Jusqu'à présent, le passage de la capitale à Los Altos avait été pour moi une sorte de retour au temps de mon enfance et à l'aube de mon adolescence, grâce à une reprise de contact avec des façons de vivre, des saveurs, des mots, des choses qui m'avaient marqué plus profondément que je ne l'aurais cru [...] Mais maintenant commençait un au-delà des images qui s'étaient présentées à mon regard quand j'avais cessé de connaitre le monde uniquement par le toucher. 4

C'est ainsi que le narrateur va remonter le cours du temps, d'abord jusqu'à la Renaissance, puis jusqu'au Moyen Âge: * Nous sommes peut-être en l'an 1540 , mais ce n'est pas certain. Les années diminuent, se diluent, s'estompent, en un recul vertigineux * (p. 238) Mais le voyage ne s'arrête pas là; il conduit aux sources mêmes du temps: «... Nous sommes en train de lire à l'envers, de droite à gauche, contre la clé de sol, en remontant aux mesures de la Genèse * (p. 244).

Cette formidable remontée dans le temps est néanmoins scandée par des temps forts qui ne vont pas sans évoquer les images initiatiques présentes dans "Rip Van Winkle *. Ainsi du passage de la porte étroite, qui donne accès au monde clos du passé, au paradis perdu, épisode dont le symbolisme, fortement teinté de sexualité, est on ne peut plus manifeste: *Près de cet arbre se dressait un corridor voûté, si bas et si étroit qu'il me parut impossible d'y introduire la pirogue. Elle s'engagea néanmoins dans ce tunnel si resserré que ses platsbords raclèrent durement un enchevêtrement de racines " (p. 214). C'est là, dans cet espace clos où "rien ne va plus * (p. 244), dans ce * monde qui [fait] reculer les limites de la vie humaine au point le plus ténébreux de cette nuit» (p. 244), que le narrateur du Partage des eaux rejoint Rip Van Winkle, les ancêtres hollandais y étant tout simplement remplacés par les premiers découvreurs espagnols; c'est d'ailleurs à ce stade qu'interviennent certains facteurs historiques et culturels susceptibles de différencier et de ramifier les expériences, conjointes sans être pour autant rigoureusement identiques, de l'américanité, comme le mythe de l'Eldorado, qui se superpose à celui du Wilderness états-unien: « Mais je suis soudain ébloui par une révélation: il n'y a aucune diffé-

4 Alejo Carpentier, Le partage des eaux, traduit de l'espagnol par René L. F. Durand, coll. « Folio „, Paris, Gallimard, 1976, p. 104. 
rence entre cette messe et celles qu'écoutèrent les conquérants de l'El Dorado dans des régions aussi lointaines. Le temps a reculé de quatre siècles. » (p. 237).

Une même volonté de renaissance et de métamorphose est néanmoins à l'œuvre, tant chez Carpentier que chez Irving, s'exprimant selon des modalités présentant de singulières analogies: «La délivrance de l'homme enchaîné, que j'associe mentalement à mon départ de "là-bas", contient implicitement un sens de résurrection, de retour des ombres, très conforme à la signification originelle du thrène, chant magique destiné à rendre la vie à un mort" (p. 291). De la même façon, dans Le partage des eaux, la métamorphose avorte finalement, le narrateur quittant la vallée du temps suspendu pour revenir à New-York, dans le but de remettre aux autorités les instruments de musique qu'il a découverts et de se procurer le matériel nécessaire pour retourner, du moins l'espère-t-il, de l'autre côté de la ligne de partage des eaux. Mais comme pour Rip Van Winkle, ce sera impossible; la route est perdue à jamais :

[L'homme] commet un jour l'erreur irréparable de redéfaire la route, croyant que l'exceptionnel peut l'être deux fois; il revient, mais il trouve les paysages changés, les point de référence effacés, tandis que ceux qui peuvent l'informer n'ont plus même visage... (p. 362)

\section{La route (perdue) d'Altamont}

La route d'Altamont (1966) n'est pas précisément un roman mais une chronique composée de quatre nouvelles distinctes: « Ma grand-mère toute-puissante », «Le vieillard et l'enfant", "Le déménagement " et "La route d'Altamont"; il n'en existe pas moins, à l'intérieur de ce recueil, une forme d'unité qu'un critique comme François Ricard relie au mouvement dialectique prenant place entre le thème de l'exil et celui du retour qui caractérise toute l'œuvre de Gabrielle Roy ${ }^{5}$ : "Le vieillard et l'enfant * et "Le déménagement " appartiennent aux œuvres dites de l'exil, tandis que «Ma

5 François Ricard, Gabrielle Roy, coll. * Ecrivains canadiens d'aujourdhui ", Montréal, Fides, 1975, p. 119-124. 
grand-mère toute-puissante * et *La route d'Altamont * se classent dans la catégorie des œuvres du retour ou de l'appartenance. On retrouve ainsi, dans la composition même de $L a$ route d'Altamont, l'idée d'un cycle traduisant une volonté de recommencement, l'attachement ressenti envers la figure maternelle ne constituant que le prélude au geste de l'arrachement, et inversement, le retour impliquant de façon logique l'exil préalable. Gabrielle Roy confronte donc son lecteur au schème * dramatique * par excellence, selon la terminologie de Gilbert Durand ${ }^{6}$ : celui du cycle, où le passé et l'avenir se rejoignent en un point précis.

On sait par ailleurs que les plaines de l'Ouest et les collines du Québec constituent les deux pôles entre lesquels se déploie l'imagination de l'espace chez Gabrielle Roy, notamment dans $L a$ route d'Altamont. C'est ainsi que l'univers de la plaine figure le côté nomade, hérité du grandpère, de la famille de Christine, la narratrice des quatre récits, tandis que la nostalgie des collines natales illustre la part sédentaire du clan, héritée celle-là de la grand-mère, et qui semble devoir être rattachée à cette tentation du repli en marge du temps, au creux des terres, dans le vallon endormi, qu'on peut déceler chez Washington Irving et Alejo Carpentier. Ces deux pôles vont entrer en conflit à plusieurs reprises. Déjà, dans la nouvelle intitulée * Ma grand-mère toute-puissante", l'aieule accuse son défunt mari, celui qu'elle surnomme «le bel aventurier», de l'avoir exilée dans l'Ouest, pour bientôt l'abandonner, en proie à ses souvenirs et à sa solitude, au milieu d'une progéniture hantée par le démon du continent: «Puis tous ceux de sa race [...] toi comme les autres, des indépendants, des indifférents, des voyageurs. ${ }^{7}$ Dans la nouvelle subséquente, intitulée "Le vieillard et l'enfant", le conflit familial est transposé dans l'univers de jeux des enfants, qui hésitent entre le couvert des arbres et l'infini de la plaine:

Car, au sortir de ce bois de chez mon oncle, aussitôt on se trouvait au bord d'une immense plaine tout ouverte et

6 Les structures anthropologiques de l'imaginaire. Introduction à l'archétypologie générale, coll. * Études", Paris, Bordas, 1981,550 p.

7 Gabrielle Roy, La route d'Altamont, coll. * 10/10 ", Montréal, Stanké, 1985, p. 30. 
presque tout entière en moissons. En sorte qu'on n'avait jamais su chez mon oncle ce qu'on aimait le mieux: le bois de trembles qui nous abritait, nous servait de cachette, nous faisait nous sentir chez nous; ou le grand pays étalé qui appelait au voyage. (p. 83)

C'est toutefois dans la dernière nouvelle du recueil, intitulée * La route d'Altamont *, que le conflit familial va se manifester avec le plus de vigueur. Cette nouvelle tire l'essentiel de sa dynamique de la tension imaginaire qui prend place entre les collines originelles et la plaine démesurée du Manitoba, tension qui traduit une méditation sur le temps et son orientation vectorielle (quels sont les rapports qui unissent le passé, le présent et le futur), et sur le dilemme de l'attachement et de l'arrachement à la mère. La narratrice, à la veille de partir pour l'Europe, fait un retour sur le conflit familial esquissé dans la première nouvelle du recueil, qui lui semble obscurément avoir à faire avec son destin personnel:

Pourtant de ce paysage laissé en arrière à l'origine de notre famille, il fut grandement question toujours, comme si persistait entre nous et les collines abandonnées une sorte de relation mystérieuse, troublante, jamais tirée au clair... Tout ce que j'en savais était peu de chose: un jour, grandpère avait aperçu en imagination - à cause des collines fermées peut-être? - une immense plaine ouverte; sur-lechamp il avait été prêt à partir; tel il était. Grand-mère, elle, aussi stable que ses collines, avait longtemps résisté. En fin de compte elle avait été vaincue. C'est presque toujours, dans une famille, le rêveur qui l'emporte. Vollà donc ce que je comprenais au sujet des collines perdues. (p. 190)

C'est donc en vertu de cette opposition entre les collines natales, enfermant le passé dans un lieu clos et protégé, et la plaine symbolisant les possibilités infinies du futur, que prend forme toute la nouvelle. Cette opposition se trouve personnifiée par la grand-mère et le grand-père de Christine; de là provient sans doute cette impression de déchirement qui envahit le lecteur, puisqu'il existe justement une déchirure qui traverse dans son entier "La route d'Altamont", dont on pourrait donner une foule d'exemples: « Par ces nuits de vent tiède et vaguement plaintif, je prenais conscience de ces deux âmes profondément divisées* (p. 215); *... Les êtres qui nous 
84

ont donné la vie continuant en nous, à travers nous, à lutter l'un contre l'autre, chacun voulant nous avoir à soi complètement * ( p. 225).

Se fondant sur cette déchirure, "La route d'Altamont * constitue en quelque sorte, pour Gabrielle Roy, la projection géographique de ses désirs et de ses nostalgies. Au départ, il y a la plaine immense et obsédante, mille fois contemplée, mille fois décrite: * Moi, j'aimais passionnément nos plaines ouvertes; je ne pensais pas avoir de patience pour ces petits pays fermés qui nous tirent en avant de ruse en ruse. " (p. 191) Cette grande plaine est le lieu de la pureté, de la vérité, voire de la révélation: * Cette absence de secret, c'était sans doute ce qui me ravissait le plus dans la plaine, ce noble visage à découvert ou, si l'on veut, tout l'infini en lui reflété, lui-même plus secret que tout autre. " (p. 191-92) À cet espace caractérisé par le gigantisme, la grandeur, la noblesse, s'oppose l'espace vaguement inquiétant et maléfique des collines, qui vient infléchir le sens de la quête, retarder l'heure de la révélation:

Je ne concevais pas, entre moi et ce rappel de l'énigme entière, ni collines, ni accident passager contre lequel eût pu buter mon regard. II me semblait qu'eût été contrarié, diminué, l'appel imprécis mais puissant que mon être en recevait vers mille possibilités du destin (p. 192).

C'est pourtant des collines que va venir la révélation: un jour, tandis qu'elles roulent en voiture dans la prairie, en empruntant le labyrinthe formé par ces petites routes de section qui plus que nulles autres semblent « mener plus loin et nulle par t» (p. 197), Christine et sa mère parviennent, comme par hasard, au cour du seul massif montueux du Manitoba méridional :

Christine, te rends-tu compte! Nous sommes dans la montagne Pimbina. Tu sais bien, cette unique chaîne de montagnes du sud du Manitoba! Toujours j'ai désiré y entrer. Ton oncle m'assurait qu'aucune route ne la pénétrait. Mais il $y$ en a une, il y en a une! Et c'est toi, chère enfant, qui l'a découverte!" ( p. 204)

Par un raccourci foudroyant, nous retrouvons l'univers de Washington Irving et d'Alejo Carpentier, notamment les thèmes et les motifs par lesquels les deux écrivains semblent 
être parvenus à cristalliser l'expérience américaine, comme le mouvement d'émancipation qui suit l'accès au pays secret, accès qui s'effectue par une porte étroite, par un chemin qu'on ne retrouve jamais. Ces thèmes et ces motifs, Gabrielle Roy va les utiliser pour exprimer sa redécouverte de l'univers maternel et la libération qui va bientôt accompagner celle-ci. La route qui mène au cœur de la montagne Pimbina passe ainsi par Altamont, un petit hameau *logé tout entier dans une crevasse parmi des sapins débiles» (p. 206). Cette crevasse joue le rôle de la faille par laquelle Rip Van Winkle avait pénétré au plus profond des monts Kaatskill. Le danger principal est de perdre cette route et de voir, comme le pauvre Rip Van Winle, s'évanouir le passé, et de se trouver en proie à une errance sans fin dans un pays inconnu. La mère de Christine ne cesse de le répéter: *Il y a, Christine, des routes que l'on perd absolument» (p. 208); «... Un jour ou l'autre, ajoutat-elle sur un ton de reproche assez piquant, tu finiras par perdre ma route d'Altamont» (p. 230); pour finalement conclure: "Je t'avais dit aussi qu'un jour tu finirais par perdre ma route d'Altamont» (p. 248). Une telle insistance est significative de l'importance du motif de la porte étroite qui donne accès à ce lieu protégé et clos dont parle d'ailleurs Pierre-Yves Petillon dans La grand-route. Espace et écriture en Amérique: *Les fictions d'Amérique n'auront jamais cessé de tourner autour de ce "point mort" niché au cœur du pays, lieu vert, isolé [...]» 8 .

C'est d'ailleurs en ce lieu secret que se produit la métamorphose, qu'on communique avec le passé pour mieux rejoindre l'avenir. C'est là que la mère de Christine va retrouver son passé: «Et comment se fait-il que l'être humain ne connaisse pas en sa vieillesse de plus grand bonheur que de retrouver en soi son jeune visage? * (p. 206). C'est encore là, dans le * petit pays secret» (p. 249) que Christine va découvrir ses racines les plus lointaines, et c'est toujours de là que la jeune fille va sortir transformée, et prête à se libérer, comme son «ancêtre* Rip Van Winkle, de la tutelle maternelle. La route qui passe par Altamont conduit ainsi au point précis où, les contradictions se trouvant brusquement abolies,

8 Pierre-Yves Petillon, La grand-route. Espace et écriture en Amérique, Paris, Seuil, 1979, p. 39. 
86

le futur rejoint le passé, tandis que la vie jaillit de la mort: * Tout cela, les pans de roc roussi, des baies rouges aux branches grêles, les feuilles écarlates jonchant le sous-bois, tout cela formait un adorable petit fouillis presque mort, et cependant quel cri vivant s'en échappait !n (p. 203).

\section{Expressions multiples d'un * romantisme * américain ?}

Les images récurrentes qu'on peut relever tant chez Washington Irving que chez Allejo Carpentier ou Gabrielle Roy (errance dans l'espace doublée d'une régression temporelle, passage difficile de la porte étroite, accès au monde clos d'un passé révolu, retour au monde moderne, perte irrémédiable des points de repère) seraient-elles symptomatiques, par-delà l'américanité qu'elles semblent exprimer, ne seraitce que par leur singulière répétition, de la permanence, en Amérique, d'une pensée teintée de romantisme? Les thèmes que nous avons dégagés rejoignent en effet les caractéristiques profondes du romantisme telles que définies par Marthe Robert dans Roman des origines et origines $d u$ roman ${ }^{9}$ : tendance à l'archaïsme, mystique de l'enfance, séjour bienheureux au sein de la nature mère, (entre autres) qui expriment la tension de l'esprit entre deux pôles complémentaires mais néanmoins inconciliables: «Le désir romantique est donc toujours partagé entre une ambition proprement prométhéenne et la communion mystique avec "l'âme du monde", qui est l'autre biais de la mégalomanie» (p. 115). Cette tension de l'esprit pourrait expliquer non seulement la déchirure profonde qui traverse les personnages des œuvres que j'ai analysées, mais aussi cette étrange valse-hésitation prenant place entre l'éden (le respect de la nature) et l'utopie (la domination de la nature) dans les imaginaires américains, depuis la poussée vers l'Ouest qui engendre, aux États-Unis, la nostalgie de la prairie perdue, jusqu'à la volonté farouche de dompter les rivières du Nord québécois, en dépit ou en vertu de l'opposition des environnementalistes et des populations autochtones qui font d'ailleurs référence au caractère sacré de la terre-mère pour justifier leur résistance,

9 Marthe Robert, Roman des origines et origines du roman, coll. « Tel «, Paris, Gallimand, 1987, 365 p. 
en passant par la conquête de l'Amazonie (sa géométrisation) et l'assassinat de Chico Mendez, sorte de bouc émissaire d'une société en voie de mutation.

C'est ainsi que les personnages mis en situation par Washington Irving, Alejo Carpentier et Gabrielle Roy figurent à fois la quintessence de l'expérience américaine et la pensée sur laquelle cette expérience se fonde: c'est la volonté "romantique" de tout recommencer, de tout reprendre à zéro, de remonter le cours du temps, qui motive et informe, pour reprendre l'expression de Marthe Robert, ces "robinsonnades* que constituent « Rip Van Winkle *, Le partage des eaux et La route d'Altamont. On retrouve en effet, dans le chef-d'œuvre de Daniel Defoe, certains des thèmes dégagés plus haut: voyage dans l'espace et dans le temps, retour au paradis perdu, critique implicite de la civilisation, doublée d'un recommencement de celle-ci:

Vagabond par défi plus que par une révolte sérieusement motivée, son voyage autour du monde n'est en réalité qu'un voyage au rebours du temps, un énorme bond en arrière vers le moment obscur où la vie et la mort sont presque indifférenciées. 10

Est-ce à dire que la fameuse américanité rejoindrait, de par le "romantisme» de ses principaux thèmes, une pensée et un imaginaire qui la dépassent et l'englobent jusqu'à la nier? Disons plutôt que l'américanité se trouve en relation nécessaire avec l'universalité; elle ne s'en distingue peut-être, en dernière instance, que par une singulière appropriation de certains motifs et de certaines images qui se cristallisent dans des œuvres littéraires déterminées.

10 Ibid., p. 136. 